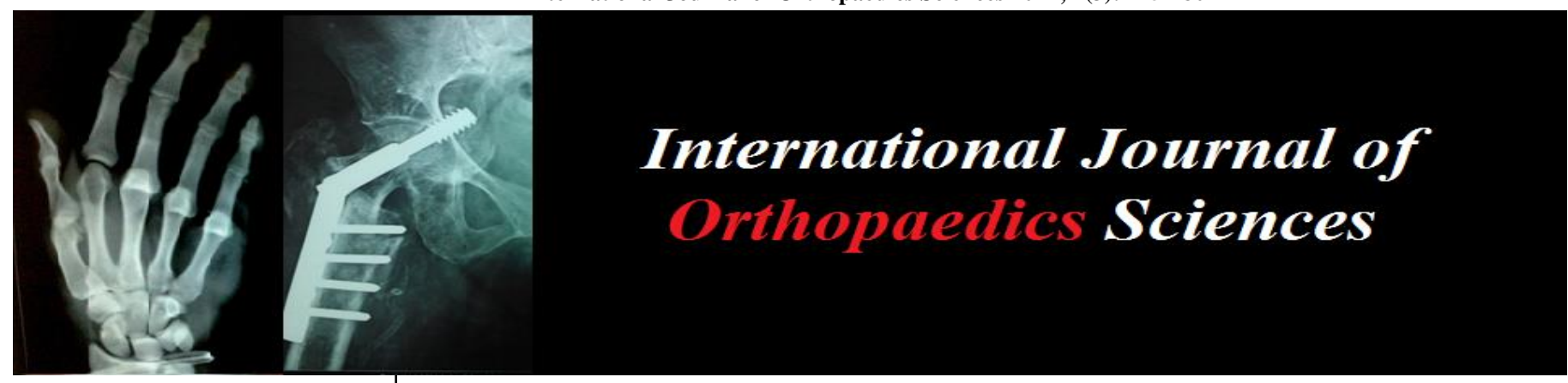

E-ISSN: 2395-1958

P-ISSN: 2706-6630

IJOS 2021; 7(3): 175-180

(C) 2021 IJOS

www.orthopaper.com

Received: 13-05-2021

Accepted: 15-06-2021

Dr. MD Qamar Abdul Azeez Associate Professor,

Department of Orthopedics, Anil Neerukonda Hospital,

Sangivalasa, Vishakhapatnam, Andhra Pradesh, India

Dr. Gompa Parameswara Rao Associate Professor,

Department of Orthopedics, Anil Neerukonda Hospital,

Sangivalasa, Vishakhapatnam,

Andhra Pradesh, India

Dr. G venkatesh

Assistant Professor,

Department of Orthopedics,

Anil Neerukonda Hospital,

Sangivalasa, Vishakhapatnam,

Andhra Pradesh, India

Dr. Chinni Ganesh

Postgraduate Resident,

Department of Orthopedics,

Anil Neerukonda Hospital,

Sangivalasa, Vishakhapatnam,

Andhra Pradesh, India

\section{A prospective study of radiological and functional outcome of non union scaphoid fractures treated by pronator quadratus muscle pedicle bone graft}

\author{
Dr. MD Qamar Abdul Azeez, Dr. Gompa Parameswara Rao, Dr. G \\ Venkatesh and Dr. Chinni Ganesh
}

DOI: https://doi.org/10.22271/ortho.2021.v7.i3c.2743

\begin{abstract}
Scaphoid fractures are common, but present unique challenges because of the particular geometry of the fractures and the tenuous vascular pattern of the scaphoid. Delays in diagnosis and inadequate treatment for acute scaphoid fractures can lead to nonunions and subsequent degenerative wrist arthritis.

Scaphoid nonunions can present with or without avascular necrosis of the proximal pole, and may show a humpback deformity on the radiograph. If left untreated, scaphoid nonunions can progress to carpal collapse and degenerative arthritis. Surgical treatment is directed at correcting the deformity with open reduction and internal fixation with bone grafting. Recently, vascularized bone grafts have gained popularity in the treatment of scaphoid nonunions, particularly in cases with avascular necrosis.

This study gives us a radiological and functional outcome of 10 cases operated by pronator quadratus based muscle pedicle bone graft for scaphoid non unions at waist level.
\end{abstract}

Keywords: scaphoid, fracture, nonunion, treatment, surgery

\section{Introduction}

The scaphoid is the most commonly fractured carpal bone, accounting for approximately $60 \%$ of all carpal fractures ${ }^{[1]}$. The importance of a correct diagnosis and appropriate treatment of scaphoid fractures lies in its blood supply. The main blood supply to the scaphoid is from the radial artery. Over $80 \%$ of the scaphoid surface is covered with articular cartilage. The dorsal scaphoid branches from the radial artery enter the nonarticular portion of the scaphoid at the dorsal ridge at the level of the waist and supply the proximal $70 \%$ to $80 \%$ of the scaphoid ${ }^{22]}$. The volar scaphoid branches from either the radial artery or the superficial palmar branch enter at the distal tubercle and supply the distal $20 \%$ to $30 \%$ of the scaphoid. Thus, the vascularity of the proximal pole depends entirely on intraosseous blood flow. This tenuous blood supply to the proximal pole of the scaphoid helps to explain the increased frequency of delayed union, nonunion and avascular necrosis (AVN) of scaphoid fractures.

AVN is reported to occur in $13 \%$ to $50 \%$ of scaphoid fractures, with an even higher incidence in those involving the proximal one-fifth of the scaphoid ${ }^{[3,4]}$.

Scaphoid fractures can escape early detection because in many cases they are subtle and the initial symptoms are minimal. Missed scaphoid fractures have a high risk of nonunion or malunion. Nonunion occurs in up to $12 \%$ of patients if an occult fracture is not detected and treated ${ }^{[3,4]}$. Scaphoid nonunions are especially challenging to treat successfully, and if untreated, they can progress to carpal collapse and degenerative arthritis. Early diagnosis and appropriate treatment of an acute scaphoid fracture and the possible consequences of nonunion are critical to avoiding debilitating wrist problems.
Corresponding Author: Dr. MD Qamar Abdul Azeez Associate Professor, Department of Orthopedics, Anil Neerukonda Hospital, Sangivalasa, Vishakhapatnam, Andhra Pradesh, India 


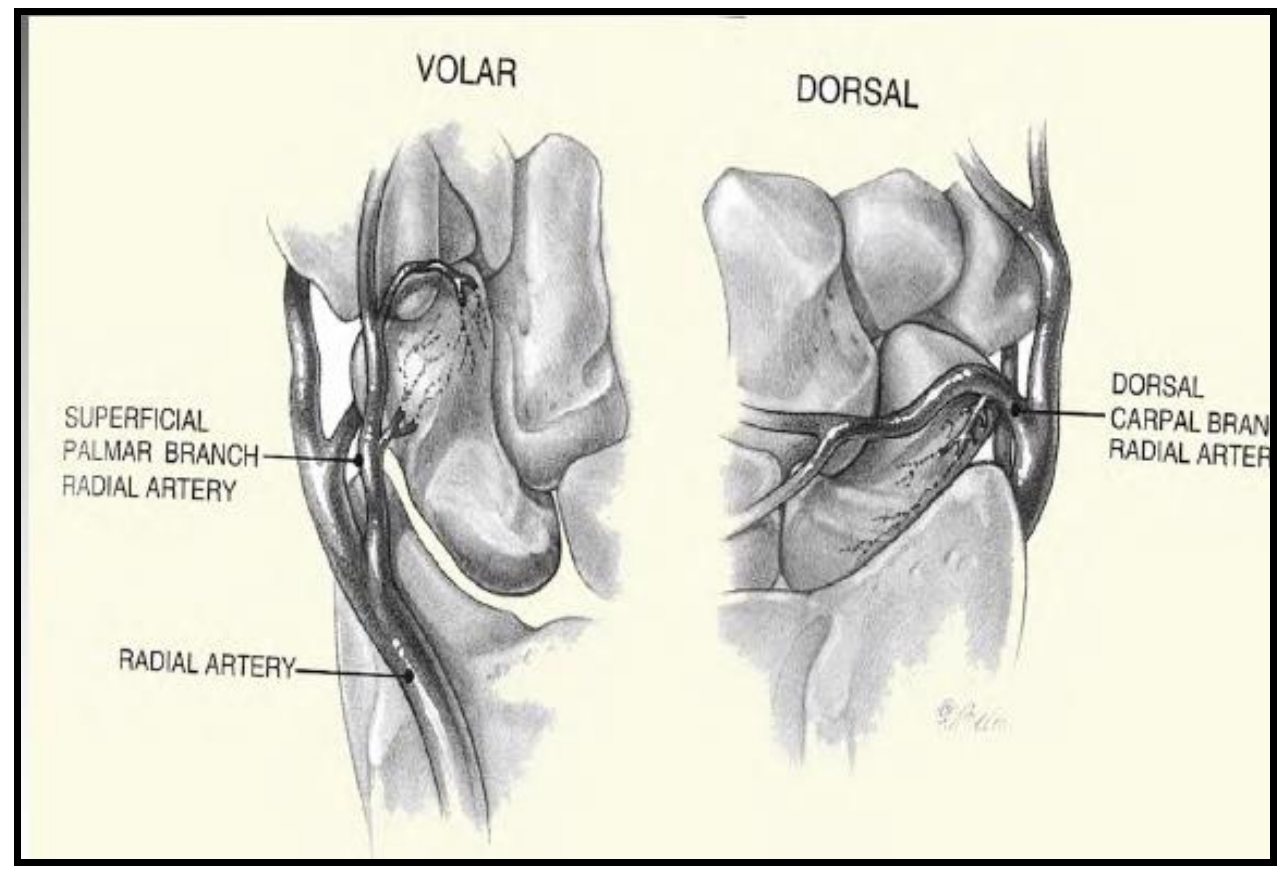

Fig 1: showing the tenuous blood supply of Scaphoid

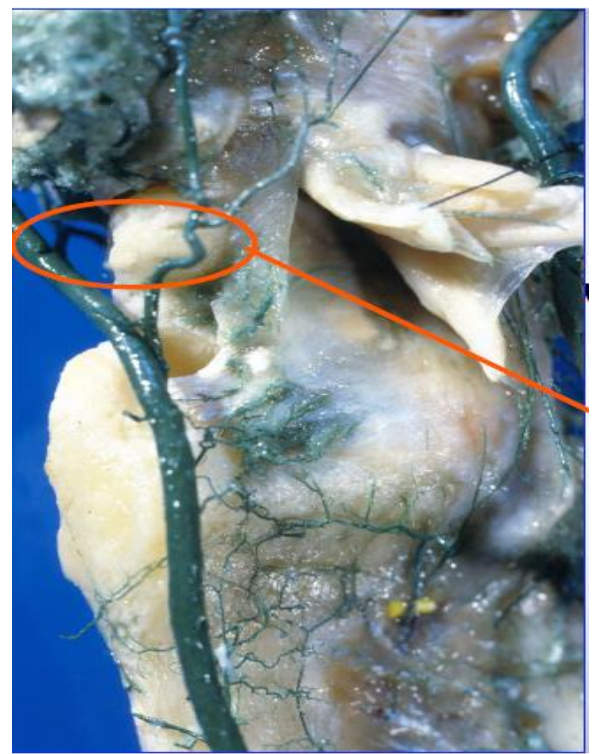

Fig 2: Retrograde supply of scaphoid from a branch of radial artery

\section{Classification}

Treatment classification system (Slade and Geissler) for scaphoid nonunion ${ }^{[5]}$

\begin{tabular}{|c|c|}
\hline I & Scaphoid fractures with delayed presentation for treatment: $4-12$ weeks \\
\hline II & Fibrous union: minimal fracture line at nonunion interface, no cyst or sclerosis \\
\hline III & Minimal sclerosis: bone resorption at nonunion interface $<1 \mathrm{~mm}$ \\
\hline IV & Cystic formation and sclerosis: bone resorption at nonunion interface $>1 \mathrm{~mm}$ but $<5 \mathrm{~mm}$, cyst, no deformity of lateral radiographs \\
\hline V & Deformity and/or pseudoarthrosis: bone resorption at nonunion interface $>5 \mathrm{~mm}$, cyst, fragment motion, deformity on lateral radiographs \\
\hline VI & Wrist arthrosis: scaphoid nonunion with radiocarpal and/or midcarpal arthrosis \\
\hline
\end{tabular}

Special circumstances

PP Proximal pole $\quad$ The proximal pole of the scaphoid has a tenuous blood supply and a mechanical disadvantage, which places it at greater nonunion risk of delayed or failed union. Because of these difficulties, this injury requires aggressive treatment to ensure successful healing.

AVN

Scaphoid nonunion with necrosis is suggested by MRI demonstrating a decrease or absence of vascularity of one or both poles. Bone biopsy can confirm necrosis. Intraoperative inspection of the scaphoid for punctate bleeding is considered definitive.

\begin{tabular}{l|l} 
LI & Ligament injury is suggested by static and dynamic imaging of the carpal bones. Arthroscopy is the most sensitive tool
\end{tabular} for detecting carpal ligament injury 
Mayo Wrist Score

\begin{tabular}{|c|c|}
\hline Section 1 - Pain Intensity & Section 2 - Functional Status \\
\hline No pain & Returned to regular employment \\
\hline Mild Occasional & Restricted employment \\
\hline Moderate, tolerable & Able to work, but unemployed \\
\hline Severe to intolerable & Unable to work because of pain \\
\hline
\end{tabular}

\begin{tabular}{|c|c|}
\hline \multicolumn{2}{|c|}{ Section 3 (choose either 3a or 3b) } \\
\hline $\begin{array}{c}\text { 3a - Range of Motion (\% of } \\
\text { normal side) }\end{array}$ & $\begin{array}{c}\text { 3b - If only injured hand } \\
\text { examined }\end{array}$ \\
\hline $100 \%$ & Greater than 120 degrees \\
\hline $75-99 \%$ & $90-120$ degrees \\
\hline $50-74 \%$ & $60-90$ degrees \\
\hline $25-49 \%$ & $30-60$ degrees \\
\hline $0-24 \%$ & less than 30 degrees \\
\hline
\end{tabular}

\begin{tabular}{|c|c|}
\hline \multicolumn{2}{|c|}{ Interpreting the Wrist Mayo Score } \\
\hline $90-100$ & Excellent \\
\hline $90-100$ & Good \\
\hline $60-90$ & Satisfactory \\
\hline Below 60 & Poor \\
\hline
\end{tabular}

\section{Materials and Methods}

A total of 10 patients with nonunion scaphoid fracture at waist level were included in our prospective study. This study was at Anil neerukonda hospital, attached to NRI institute of medical sciences, Visakhapatnam, Andhra Pradesh, India between October 2018 and March 2020. Written informed consent was obtained from all subjects prior to the study. All subjects are between the ages 25 and 40 years. Subjects with a history of systemic disorders affecting the musculoskeletal system, bone tumors, or bone infection were excluded. We also excluded subjects with a simultaneous fracture of other wrist bones, apparent degenerative changes in wrist bones, or radiologically proven scapholunate dissociation from the study.

We evaluated all subjects with standard radiographs of both wrists with postero-anterior views in neutral rotation, lateral views and special scaphoid views. The presence of an avascular proximal pole, dorsal intercalated segmental instability, radioscaphoid arthritis or dorsal osteophytes was noted. MR imaging was done for all subjects for the assessment of the vascularity of the proximal pole.

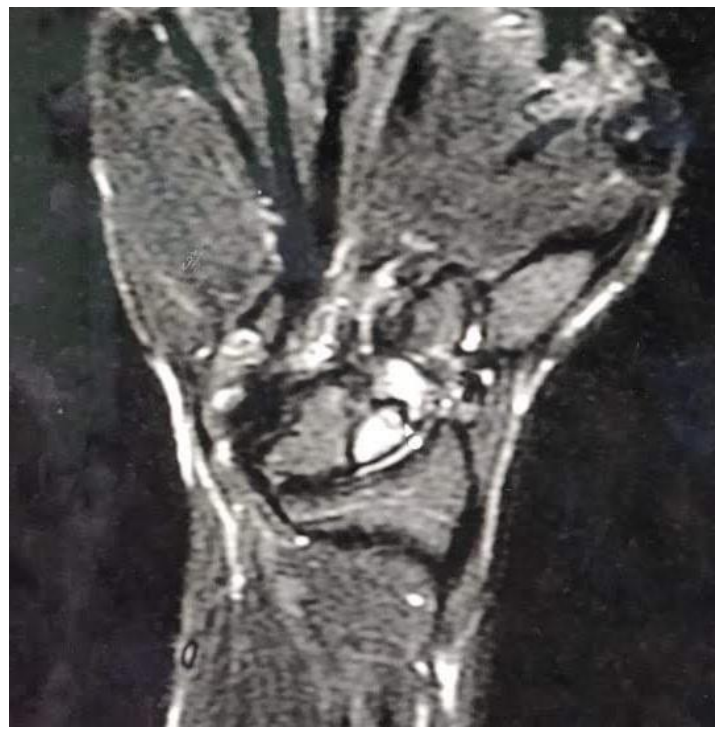

Preoperative MRI showing Cystic degenerative changes along with Nonunion of scaphoid fracture

\section{Surgical Technique}

The surgery was done through a palmar approach ( Extended volar approach) for all subjects.Once capsulotomy was done and scaphoid exposed the proximal and distal parts of the bone were curetted of sclerotic bone and fibrous tissue at the nonunion site using a dental burr. Then a small square block of bone was marked at the insertion of Pro Quad muscle on the radius and was elevated from distal border of the muscle taking $25 \%$ of muscle belly.Through temporary overdistraction facilitated by the joystick K-wires, the graft is interposed at the nonunion site, with particular care to correct the length and the rotational malalignment of the nonunited scaphoid. The bone graft was then secured with two $0.8 \mathrm{~mm}$ Kwires, one of which extends into the lunate. Under fluoroscopic control in two planes the optimal alignment of the scaphoid was ensured. Once satisfactory reduction was ascertained, length of the screw was calculated and drilled appropriately over a guide wire and Herbert screw was placed.

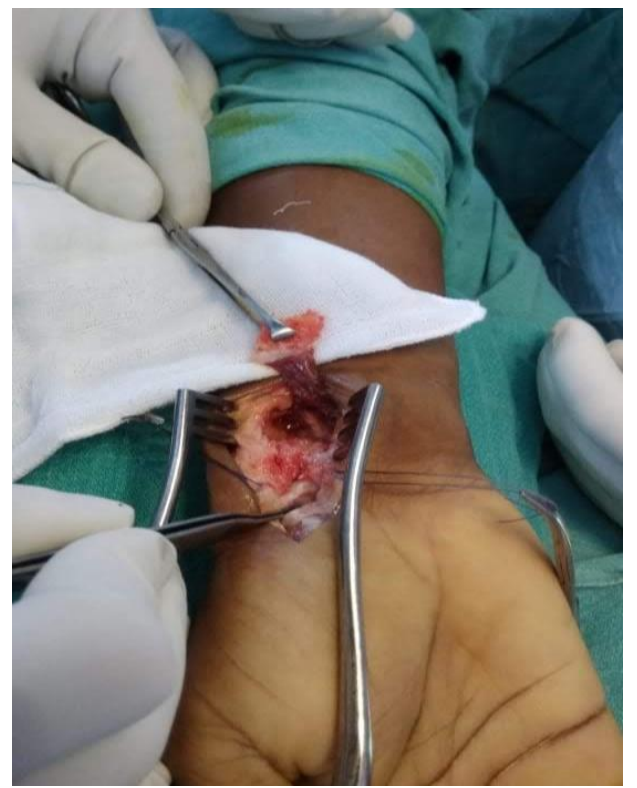

Fig 3a: Surgeon holding the square bone block with attached pronator quadratus muscle belly harvested from volar distal end radius

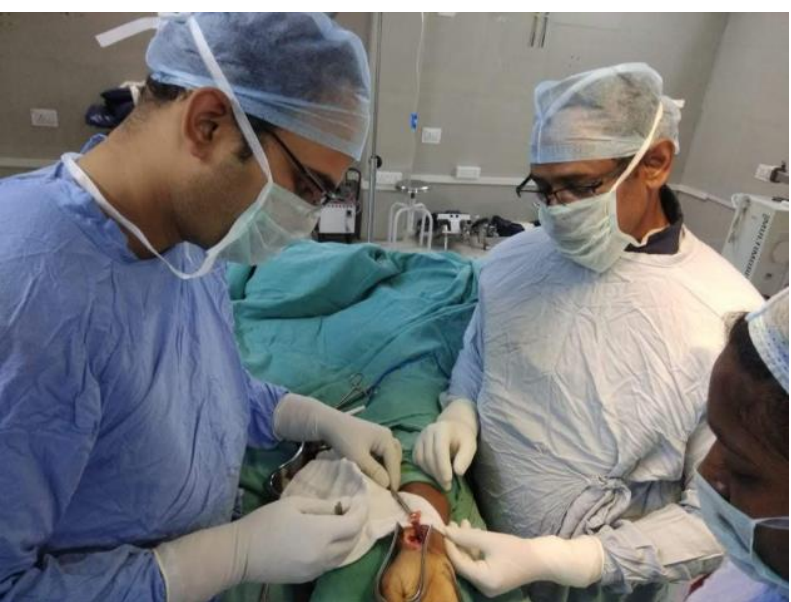

Fig 3b: Surgeon holding the square bone block with attached pronator quadratus muscle belly harvested from volar distal end radius 


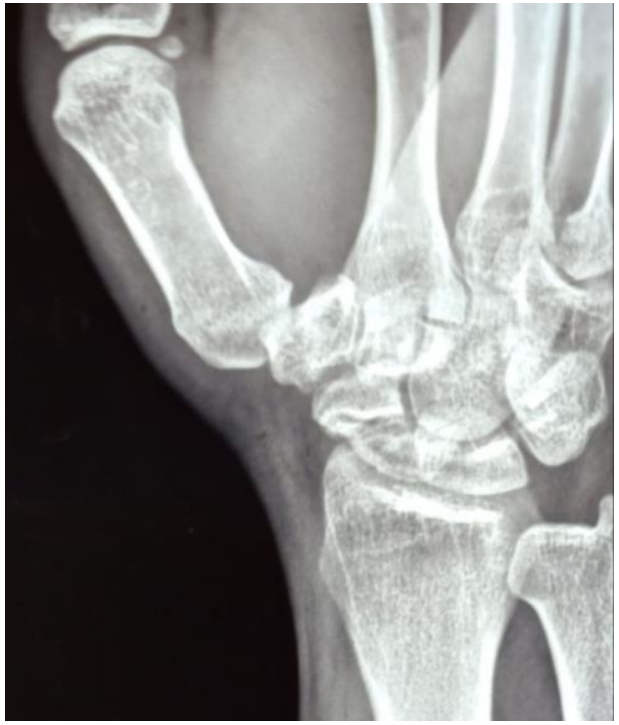

Fig 4: Pre Op AP Xray showing nonunion waist fracture of scaphoid

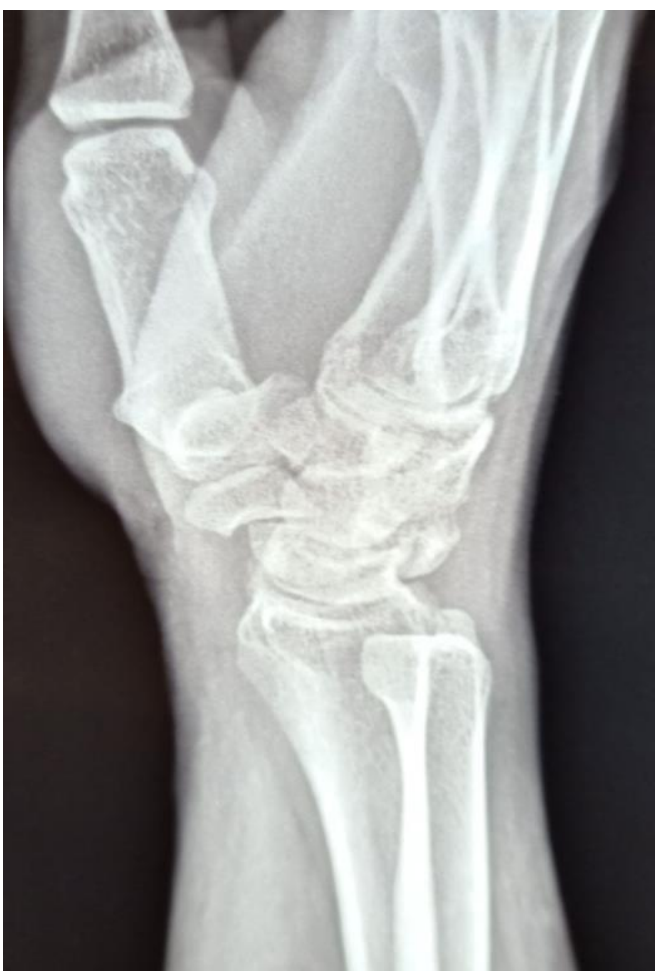

Fig 5: Pre Op Lateral xray of nonunion waist fracture of scaphoid

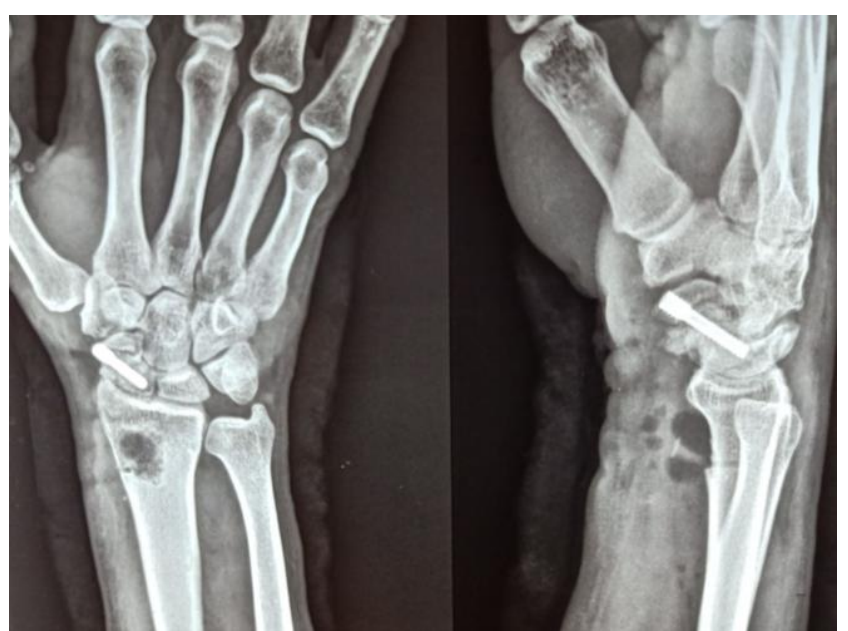

Fig 6: Immediate Post op Xray showing Graft packed at fracture non union site and stabilized with herbert screw.

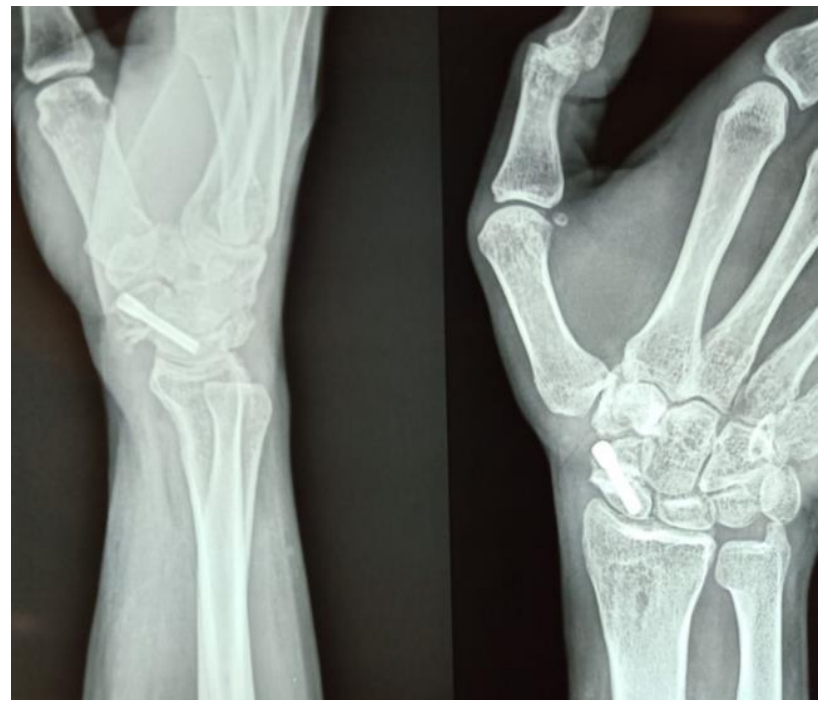

Fig 7: 6th month follow up xray showing donor site healed state and fracture progressive union features.

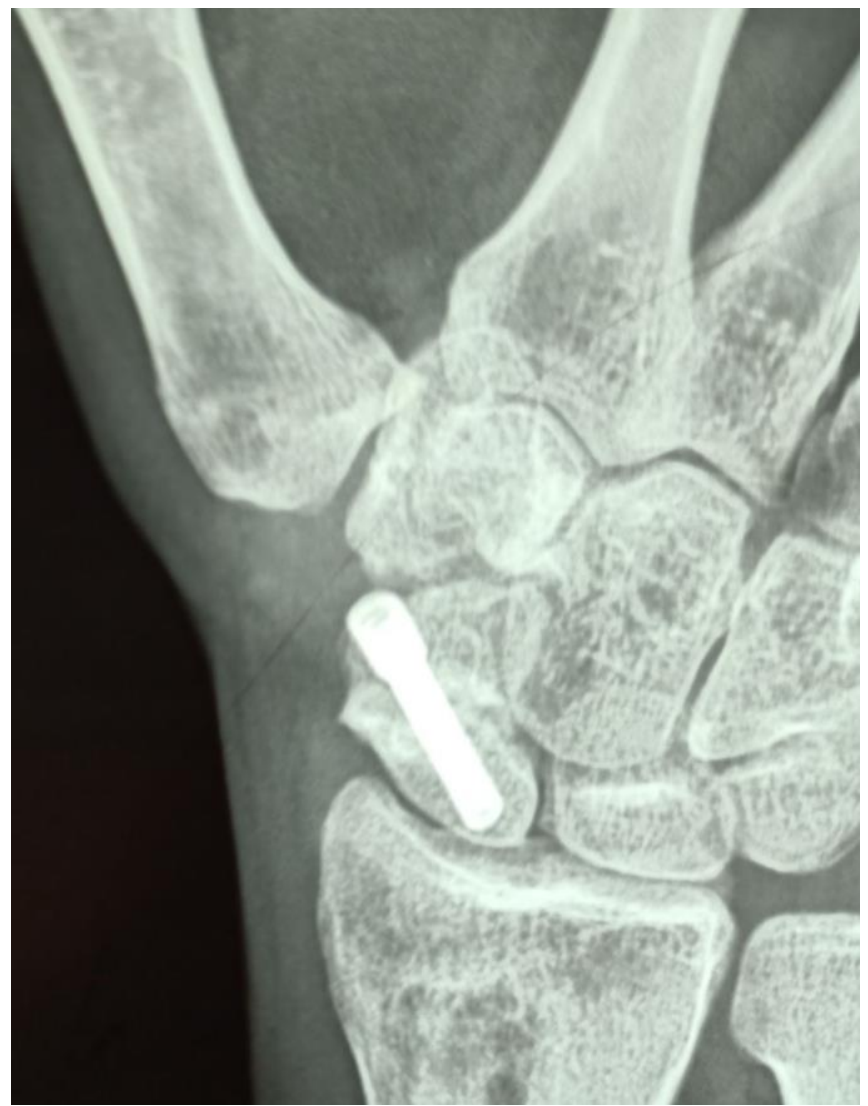

Fig 8: 9th month follow up showing continuity of trabecular pattern.

Postoperative care A long arm cast with thumb spica is applied postoperatively. The $\mathrm{K}$-wires are removed 5 weeks after the procedure and the hand is immobilized in a short arm cast for an additional period of $2-5$ weeks

The union was considered to have occurred when there was no tenderness at the anatomical snuffbox and there was evidence of bony trabeculae crossing the fracture line at the site of the fracture on at least three views. Mean time to radiological union for all fractures is Ten weeks. Thus, we evaluated the union in patients four and six months after surgery, to ensure that the surgery had its effect on the patient. The patients were assessed based on the Mayo wrist score (MWS) questionnaire on a follow-up after six months. The questionnaire consists of questions assessing pain intensity, functional status, range of motion, and grip strength. Scores 
are recorded in four groups: 90- 100: Excellent, 80-90: Good, 60-80: Satisfactory, below 60: poor.

ROM in dorsiexion, palmar exion, radial exion, and ulnar exion in the affected wrist was measured using a goniometer, and was reported as a percentage. Grip strength was measured by asking the patient to squeeze the index finger of the examiner, and the strength was compared on the contralateral side.

\section{Results}

A total of 10 patients with nonunion scaphoid fracture were evaluated in this study. Mean age of the subjects was $21.08 \pm$ 6.09 years ranging from 25 to 40 years. Twenty four weeks after surgery, fracture in all 10 patients was united.

Sixmonths after surgery, mean Mayo Wrist Score was $73.0 \pm$ 8.36 in all the subjects, ranging from 65 to 85 . Two subjects $(20 \%)$ were grouped as excellent, $4(40 \%)$ good and 4 $(40.0 \%)$ satisfactory.

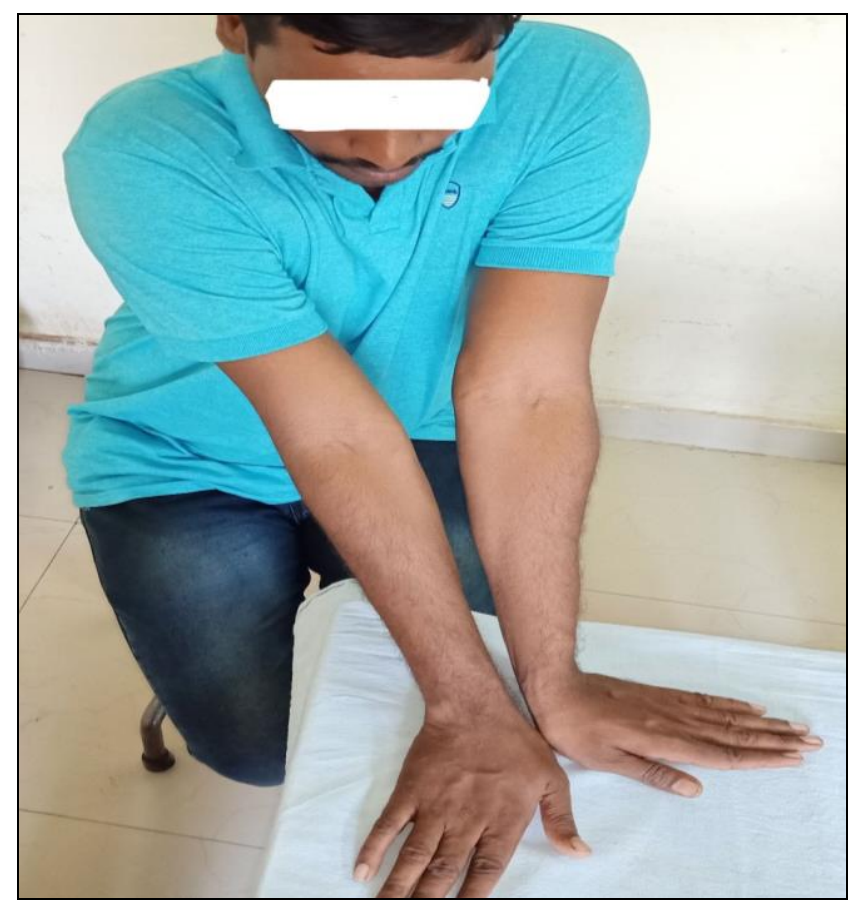

Fig 9a: 6th month followup

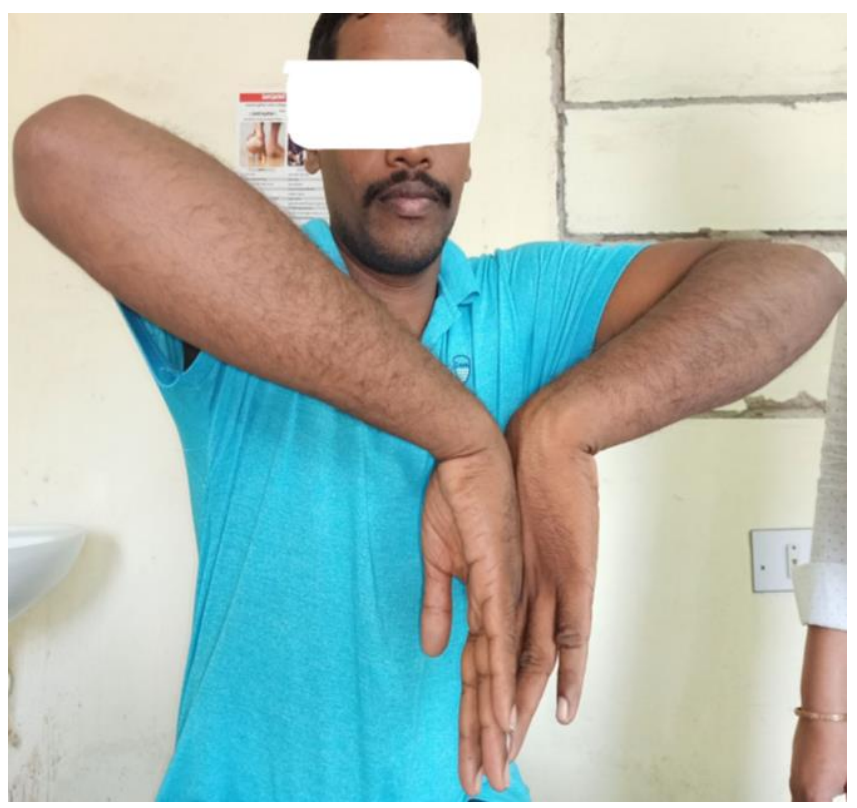

Fig 9b: 6th month followup

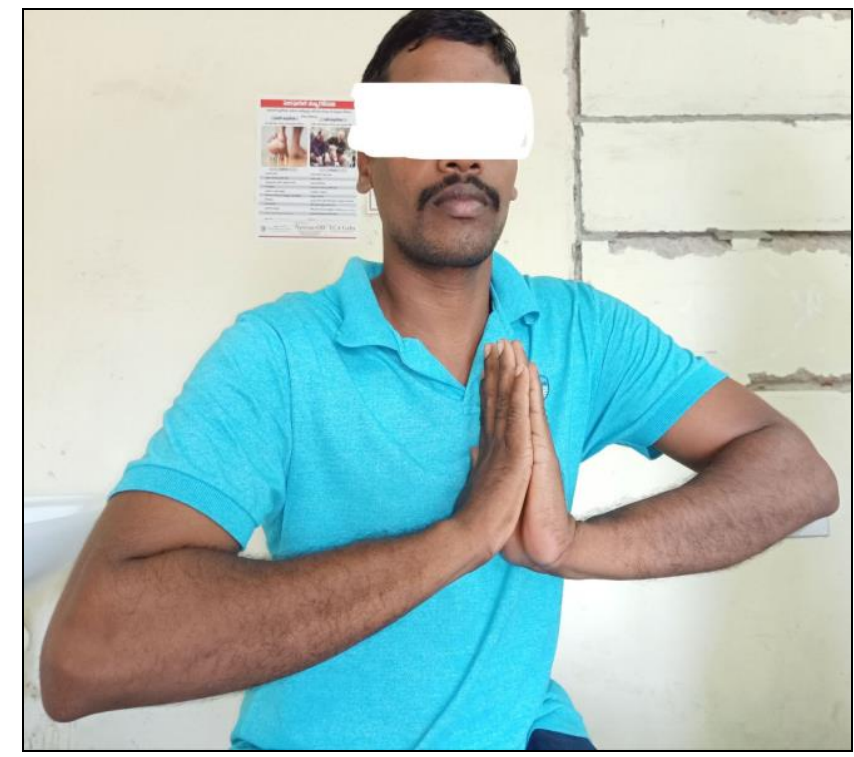

Fig 9c: 6th month followup

\section{Discussion}

In this study, we evaluated the clinico radiological outcome of 10 patients with scaphoid fracture nonunion at waist level operated with pronator quadratus based muscle pedicle bone graft. The results were satisfactory based on the Mayo wrist score questionnaire.

The meticulous surgical technique and the attention to detail are the prerequisites for the success of the procedure and the avoidance of potential pitfalls.

\section{Several technical points are underlined in the present report.}

- The cessation of smoking (pre- and postoperatively) to avoid its undesirable effect on the bone healing process and the vascularity.

- The tourniquet application simply with forearm elevation, avoiding Esmarch's bandage exsanguination so that the small vessels of the distal radius will remain visible.

- The surgeon needs to be familiar with the microvascular anatomy of the distal radius

- The preoperative planning of the graft dimensions comparing radiographs of both wrists, and the intraoperative shaping and size matching of the graft to increase the length of the scaphoid and correct the 'humpback' deformity and the height of the carpus (2-4)

- The interposition of the graft in full width as an intercalary bone between the two segments of the scaphoid, and not as an inlay graft bridging the nonunion. The difficulty of the correction of the 'humpback' deformity and of the application of the graft can be addressed by using K-wires as joysticks in each scaphoid segment

The management of scaphoid nonunions with pedicled vascularized bone grafts from the distal radius, although technically demanding, results in a high union rate as a viable graft with its intrinsic vascularity preserved is interposed between the scaphoid segments, in revascularization of an avascular proximal pole, and in correction of the 'humpback' deformity leading also to the carpal alignment.

\section{Conclusions}

This study shows that the muscle pedicle bone graft with pronator quadratus muscle, although old, is still a reliable and 
inexpensive method for the treatment of scaphoid bone nonunion, showing high percentages of healing and functional improvement, and it can be used in line with the new vascularized surgery methods.

\section{References}

1. Dailiana ZH, Malizos KN, Urbaniak JR. Vascularized periosteal flaps of distal forearm and hand. J Trauma 2005;58:76-82.

2. Malizos KN, Dailiana ZH, Kirou M, Vragalas V, Xenakis TA, Soucacos PN. Longstanding nonunions of scaphoid fractures with bone loss: successful reconstruction with vascularized bone grafts. J Hand Surg [Br] 2001;26:330334.

3. Malizos $\mathrm{KN}$, Zachos $\mathrm{V}$, Dailiana $\mathrm{ZH}$, Zalavras $\mathrm{C}$, Varitimidis S, Hantes M, Karantanas A. Scaphoid nonunions management with vascularized bone grafts from the distal radius: a clinical and functional outcome study. Plast Reconstr Surg 2007;119:1513-1525.

4. Zaidemberg C, Siebert JW, Angrigiani C. A new vascularized bone graft for scaphoid nonunion. J Hand Surg [Am] 1991;16:474-478.

5. Slade JF3rd, Merrell GA, Geissler WB, Geissler WB. Fixation of acute and selected nonunion scaphoid fractures. Wrist Arthroscopy Springer, New York, NY 2005, 112-24. 